\title{
Design of Fuzzy Neural Network Guidance Law Based on Takagi- Sugeno for UAV
}

\author{
${ }^{1}$ Yibo Li, ${ }^{2}$ Zitong Li, ${ }^{3}$ Yi Wang and ${ }^{4}$ Wei Chen \\ ${ }^{1,2}$ School of Automation, Shenyang Aerospace University, Liao Ning, China \\ AVIC Chengdu Aircraft Industrial (group) CO., LTD, Chengdu, China \\ 1liyibo_sau@163.com, 22itong19890903@163.com, 3scjjwy@21cn.com, \\ ${ }^{4}$ scjjwy@21cn.com
}

\begin{abstract}
Guidance of unmanned aerial vehicle (UAV) in three-dimensional space was studied in this paper. We designed a three-dimensional fuzzy neural network guidance law with the fuzzy rules of Takagi-Sugeno that based on proportional guidance principle and put the characteristics of the fuzzy neural network control into use. We decomposed the 3-D tracking trajectory into two two-dimensional planes and guided respectively, and took the influence of target mobility for guidance trajectory into consider. The design of guidance law was using the principle of proportional guidance, based on the Takagi-Sugeno type fuzzy inference, we took horizontal line of approaching velocity of missile-to-target, vertical line of sight angle velocity and sight angle velocity as inputs of fuzzy neural network, and yaw and pitch acceleration commands of UAV are the outputs, then we simulated the seeker tracking process against the target doing uniform motion in a straight line, curve movement and uniform circular motion respectively for UAV. Verified by simulation in this paper, the effect of the design of guidance law is ideal, and the proposed law is superior to the others and has good effect in response to the maneuvering target, and guidance trajectory is smoother.
\end{abstract}

Keywords: proportional guidance, fuzzy neural network, Difference equation, TakagiSugeno

\section{Introduction}

In recent years, UAV has rapidly developed from the traditional single effects like surveillance, reconnaissance and other combating support role towards the direction of the attack, becoming a means of implementing air attracting precisely [1]. And the guidance of UAV is different from the missile guidance; the guidance of missile focuses on getting smaller terminal miss-distance and overloads which conform to the limit. But the purpose of UAV guidance just need to guidance the UAV to the area where meet the attacking conditions, so as to provide necessary conditions for effective attack. So the design of UAV guidance law is the key for UAV to implement effective tracking and intercepting against the target in air war [2].

Guidance methods have developed from the initial proportion guidance, tracking method, the parallel approaching method to the intelligent guidance, such as: fuzzy guidance, the neural network guidance, etc., [3-8]. And Takagi-Sugeno fuzzy inference with its characteristics of simple calculation, good for mathematical analysis, and combinating with PID and other optimization adaptive methods easily is widely used, such as the literature [9] used the T-S fuzzy neural network to realize the higher precision prediction of traffic flow. Literature [10] used the T-S fuzzy neural network to realize the quick and efficient fault 
diagnosis of circuit, etc. Fuzzy neural network is the combination of neural network and fuzzy unit, it does not rely on the mathematical model of controlled object, it can automatically adjust its rules according to the change of controlled object, so it get much attention at present. Such as [11-14], they all did research on fuzzy neural network and applied it to the practice, such as water quality evaluation, etc.

At present, most of the literatures only gave the guidance methods that based on fuzzy or neural network. So in the process of research in this paper, we applied the guidance to UAV, at the same time expanded the research into three-dimensional space, and then we introduced fuzzy neural network that based on Takagi-Sugeno fuzzy rules to simulate. During the simulation, we put the difference equations into use, then decomposed the tracking process into two planes: horizontal and vertical surface, The design of the guidance law was based on T-S fuzzy logic control method, and we took horizontal line of sight angle as the input of fuzzy logic controller by using the proportional guidance, the output is the acceleration of UAV, so as to control the rate of the horizontal line of sight angle in the range that is close to zero. In order to control the UAV approaching the target with the fastest speed, the approaching velocity of UAV-to-target is as another input. Every once in a while interval, according to the 3-d coordinate we can draw up the guidance trajectory. Verified by simulation in this paper, the effect of the design of guidance law is ideal, and the proposed law is superior to the others and has good effect in response to the maneuvering target, and guidance trajectory is smoother.

\section{3-Dimensional Pursuit-Evasion Model}

In this paper we adopted the method described in literature [15] to build the threedimensional model what is shown in Figure 1.

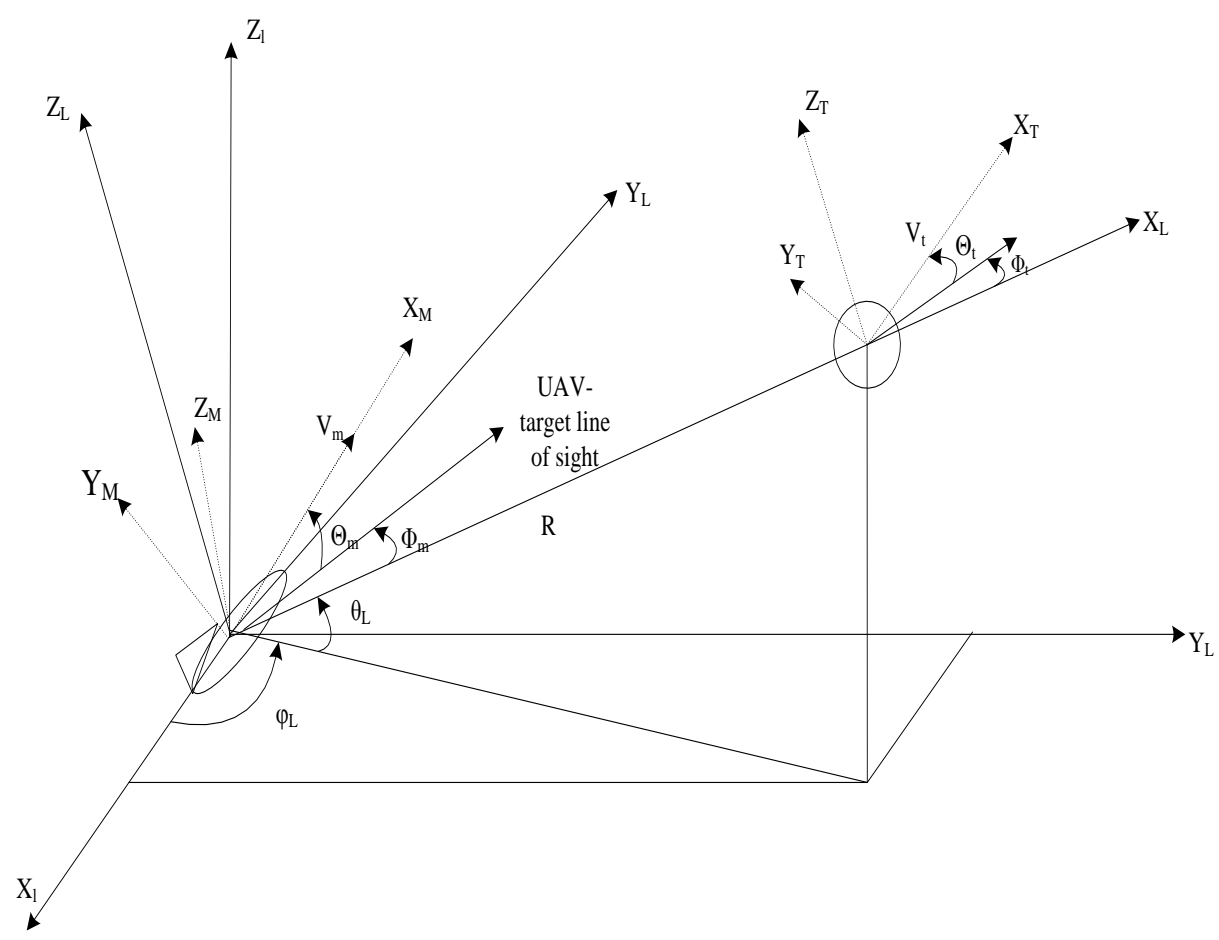

Figure 1. 3-dimensional Pursuit-evasion Model 
In Figure 1, $\left(\mathrm{OX}_{1} Y_{l} Z_{l}\right)$ - Reference (ground) coordinate system;

$\left(O X_{L} Y_{L} Z_{L}\right)$ - Line of Sight (LOS) coordinate system;

$\left(O X_{M} Y_{M} Z_{M}\right)$ - UAV body coordinate system;

$\left(O X_{T} Y_{T} Z_{T}\right)$ - Target body coordinate system, where the meanings of all notations has been already described in reference.

There are four coordinate systems existing in 3-dimensional pursuit-evasion model. Seen from Figure 1, we can know that the transformation matrix $\mathrm{L}_{\mathrm{ML}}$ of $\mathrm{UAV}$ body coordinate system and the line of sight coordinate system, the transformation matrix $L_{T L}$ of the target body coordinate system and the line of sight coordinate system are shown respectively as following formulas:

$$
\begin{aligned}
\mathrm{L}_{M L}= & {\left[\begin{array}{ccc}
\cos \varphi_{M} & -\sin \varphi_{M} & 0 \\
\sin \varphi_{M} & \cos \varphi_{M} & 0 \\
0 & 0 & 1
\end{array}\right]\left[\begin{array}{ccc}
\cos \theta_{M} & 0 & -\sin \theta_{M} \\
0 & 1 & 0 \\
\sin \theta_{M} & 0 & \cos \theta_{\mathrm{M}}
\end{array}\right] } \\
= & {\left[\begin{array}{cccc}
\cos \theta_{M} \cos \varphi_{M} & -\sin \varphi_{M} & -\sin \theta_{M} \cos \varphi_{M} \\
\cos \theta_{M} \sin \varphi_{M} & \cos \varphi_{M} & -\sin \theta_{M} \sin \varphi_{M} \\
\sin \theta_{M} & 0 & \cos \theta_{M}
\end{array}\right] } \\
L_{T L} & {\left[\begin{array}{cccc}
-\cos \varphi_{T} & -\sin \varphi_{T} & 0 \\
\sin \varphi_{T} & -\cos \varphi_{T} & 0 \\
0 & 0 & 1
\end{array}\right]\left[\begin{array}{ccc}
\cos \theta_{T} & 0 & -\sin \theta_{T} \\
0 & 1 & 0 \\
\sin \theta_{T} & 0 & \cos \theta_{T}
\end{array}\right] } \\
= & {\left[\begin{array}{ccc}
-\cos \theta_{T} \cos \varphi_{T} & -\sin \varphi_{T} & \sin \theta_{T} \cos \varphi_{T} \\
\cos \theta_{T} \sin \varphi_{T} & -\cos \varphi_{T} & -\sin \theta_{T} \sin \varphi_{T} \\
\sin \theta_{T} & 0 & \cos \theta_{T}
\end{array}\right] }
\end{aligned}
$$

On the basis of literature $[16,17]$, using the formulas $(1,2)$, we transform the relevant amounts to the scalar form in the line of sight coordinate system, the engagement geometry equations describing the UAV-target's relative motion are presented as follows:

$$
\begin{aligned}
& \dot{R}=\left(\rho \cos \theta_{\mathrm{T}} \cos \phi_{T}-\sin \theta_{M} \cos \phi_{M}\right) V_{m} \\
& \dot{\theta_{M}}=\frac{a_{z m}}{V_{M}}-\dot{\phi}_{L} \sin \theta_{L} \sin \phi_{M}-\dot{\theta}_{L} \cos \phi_{\mathrm{M}} \\
& \dot{\phi_{M}}=\frac{a_{y m}}{V_{M}}+\dot{\phi}_{L} \sin \theta_{L} \cos \phi_{M} \tan \theta_{M}-\dot{\theta}_{L} \sin \phi_{M} \tan \theta_{M}-\dot{\phi}_{L} \cos \theta_{L} \\
& \dot{\theta_{L}}=\frac{\left(\rho \sin \theta_{T}-\sin \theta_{M}\right) V_{M}}{R} \\
& \dot{\phi_{L}}=\frac{\left(\rho \cos \theta_{T} \sin \phi_{T}-\cos \theta_{M} \sin \phi_{M}\right) V_{M}}{R \cos \theta_{L}} \\
& \dot{\theta_{T}}=\frac{a_{z t}}{\rho V_{T}}-\dot{\phi}_{L} \sin \theta_{L} \sin \phi_{T}-\dot{\theta}_{L} \cos \phi
\end{aligned}
$$


$\dot{\phi}_{T}=\frac{a_{y t}}{V_{T} \cos \theta_{T}}+\dot{\phi}_{L} \sin \theta_{L} \cos \phi_{T} \tan \theta_{T}-\dot{\theta}_{L} \sin \phi_{T} \tan \theta_{T}-\dot{\phi}_{L} \cos \theta_{L}$

Where, $\dot{\mathrm{R}}$ is the approaching velocity of UAV-to-target, $\phi_{\mathrm{M}}, \theta_{M}$ are the yaw angle and the pitch angle of UAV respectively. $\phi_{T}, \theta_{T}$ Are the yaw angle and the pitch angle of target respectively. $\phi_{L}, \theta_{L}$ are the yaw angle and the pitch angle of LOS that based on LOS coordinate system.

\section{The Design of Neural Network Guidance Law in 3-Dimensional Space}

The pitch and yaw acceleration of proportional guidance in 3-dimensional is shown in the following:

$$
\begin{aligned}
& a_{y m}^{c}=N V_{m} \dot{\theta}_{L} \sin \phi_{m} \sin \theta_{m}+N V_{m} \dot{\phi}_{L} \cos \phi_{m} \cos \theta_{m} \\
& a_{z m}^{c}=N V_{m} \dot{\theta}_{L} \cos \phi_{m}
\end{aligned}
$$

Seen from the formulas above, yaw acceleration a $\mathrm{ym}_{\mathrm{m}}$ can be affected by $\dot{\phi}_{\mathrm{L}}$ 和 $\dot{\theta}_{\mathrm{L}}$.

In this paper, we adapt the two inputs and single outputs fuzzy neural network guidance which based on Takagi-Sugeno fuzzy rules; it is combined by neural network and fuzzy theory.

The principle diagram of 3-dimensional fuzzy guidance control system principle diagram is as shown in Figure 2 as follows:

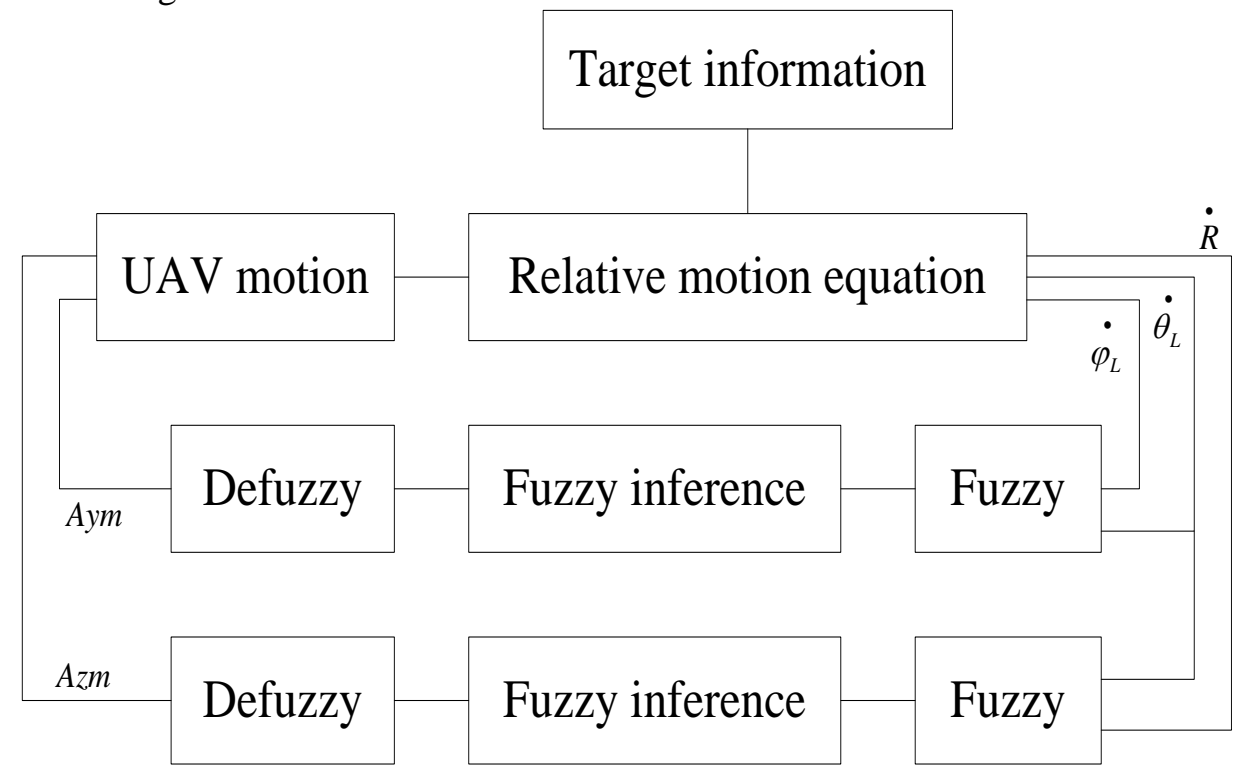

Figure 2. Fuzzy Neural Network Control Principle Diagram

Take the fuzzy neural network structure chart of Azm for instance, what is shown in Figure 3 as follows: 


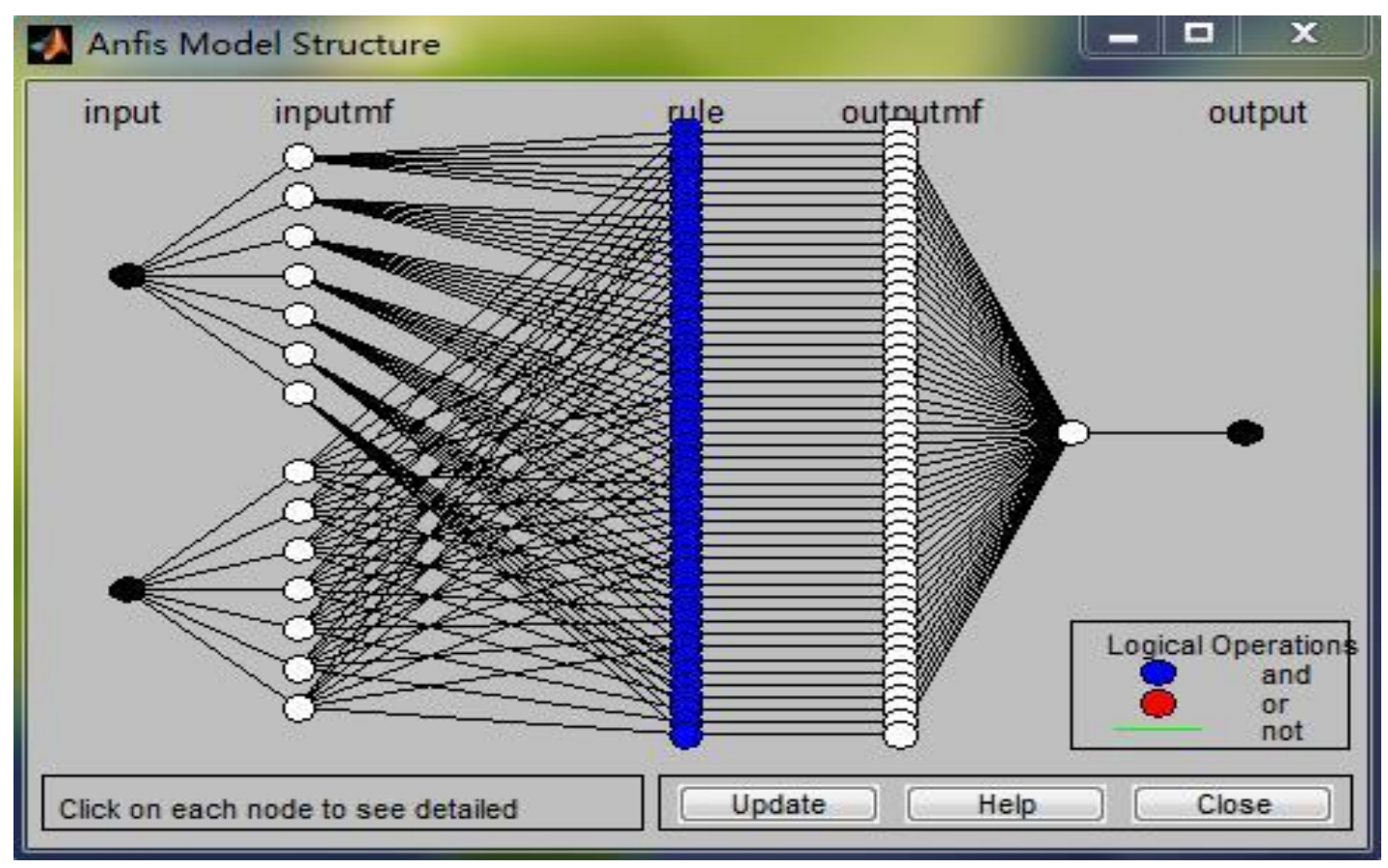

Figure 3. Fuzzy Neural Network Structure Chart of Azm

On the basis of literature [17-18], the first layer is the input layer, whose role is to deliver the input values to the next layer which is the input of fuzzy neural network.

The second layer: conditions of layer (also called fuzzy layer). Each node in the second layer represents a language variable value, such as PS, NS etc. It plays an important role in calculating the membership function of each input component belonging to which linguistic variable value of fuzzy set. The fuzzy set of $\dot{R}, \dot{\phi}_{L}, \dot{\theta}_{L}$, aym, azm are all [NB, NM, NS, ZE, PS, PM, PB]. In this paper we use Gaussian function.

The third layer: in the third layer, each node represents a piece of fuzzy rule, whose effect is to match the premise of fuzzy rules and to calculate the fitness of each rule. Fuzzy logic is the logic of "and".

In this thesis, because the control of the two planes is asymmetric, so for Aym and Azm, we need establish fuzzy controller respectively, corresponding we also need two fuzzy rule bases. There are 49 fuzzy rules in total, the model rules are as follows:

If ( $\mathrm{dR}$ is $\mathrm{NB}$ ) and (dthetaL is $\mathrm{PB}$ ) then (azm=NB);

When $\mathrm{dR}$ is "Negative Big" and dthetaL is "Positive Big", it is right on $\mathrm{X}$ axis of UAV, but the speed on $Z$ axis is not enough, so we need set Azm to be "Negative Big" to increase the rate along $\mathrm{Z}$ axis direction of UAV .

The output characteristic surface figure of fuzzy inference system of aym, azm are shown in Figure 4, Figure 6: 


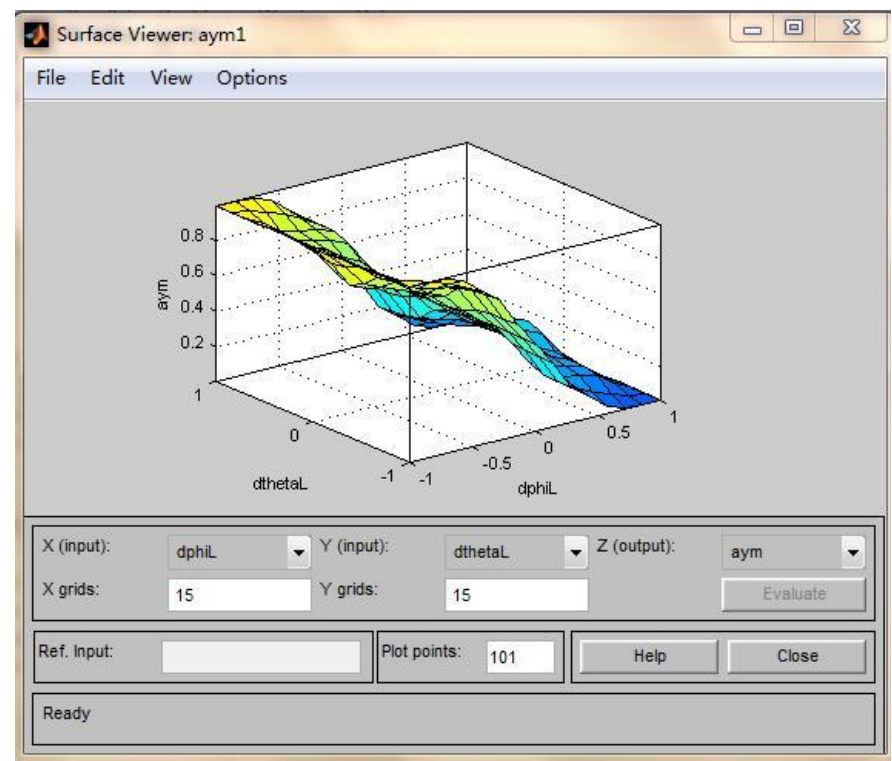

Figure 4. The Output Characteristic Surface Figure of Fuzzy Inference System of Aym

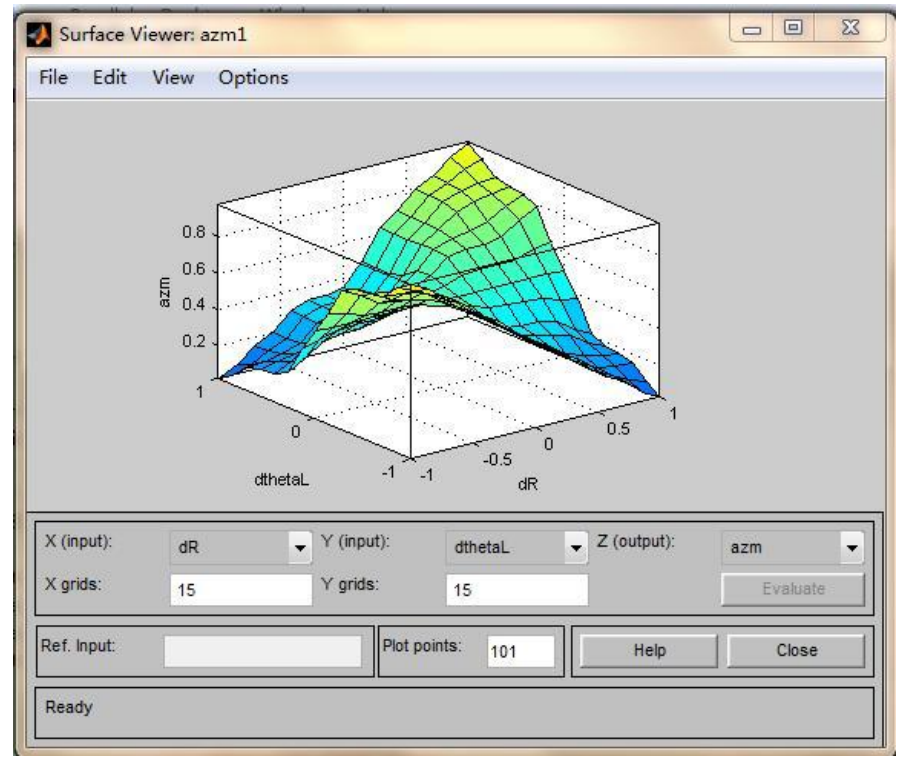

Figure 5. The Output Characteristic Surface Figure of Fuzzy Inference System of Azm

The fuzzy rule base of Azm has the effect for UAV to track targets fast, and make the pitching Angle rate as small as possible. Aym fuzzy rule base is to fix $\phi_{\mathrm{L}}$.

The second layer: the fourth layer is the function layer (Defuzzy layer); the node number is same to the third layer, which is used to implement the normalized calculation.

Layer 5 is the output layer, which is to realize the defuzzy. The method of defuzzy is wtaver [13].

So, we had established a complete fuzzy neural network controller. 


\section{Simulation Analysis}

\subsection{Simulation Parameter Settings}

In order to verify guidance effect of fuzzy neural network that based on Takagi - Sugeno fuzzy rules, we compared it with the proportional guidance. We assumed that the initial distance between the UAV and the target is 30000 meters, UAV velocity $\mathrm{Vm}=700 \mathrm{~m} / \mathrm{s}$, target velocity $\mathrm{Vt}=300 \mathrm{~m} / \mathrm{s}$, the initial yaw angle and the pitch angle of UAV is $\phi_{M}=p i / 18, \theta_{M}=-p i / 18$, the initial yaw angle and the pitch angle of target $\phi_{\mathrm{T}}=p i / 9, \theta_{T}=p i / 10 . \phi_{L}, \theta_{L}$ are the yaw angle and the pitch angle of LOS that based on LOS coordinate system, their initial values are $\phi_{L}=p i / 6, \theta_{L}=-p i / 6$. The parameters $\mathrm{N}$ of the traditional 3D proportional navigation guidance law were chosen: $\mathrm{N}=5$; When the distance between UAV and the target is less than 600 meters, the simulation ended.

\subsection{Simulation Result}

1) When the target is motionless, guidance trajectories of fuzzy neural network guidance law based on Takagi-Sugeno for UAV is shown in Figure 6 as follows:

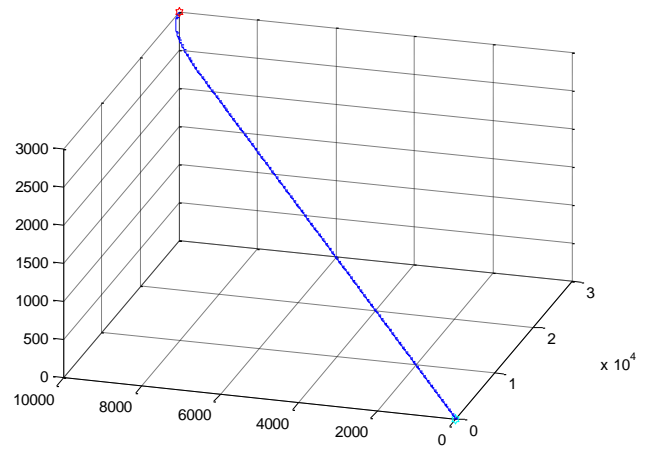

Figure 6. Trajectory of UAV Tracking Motionless Target

2) When the target is moving in line, guidance trajectories of fuzzy neural network guidance law based on Takagi-Sugeno for UAV is shown in Figure 7 as follows:

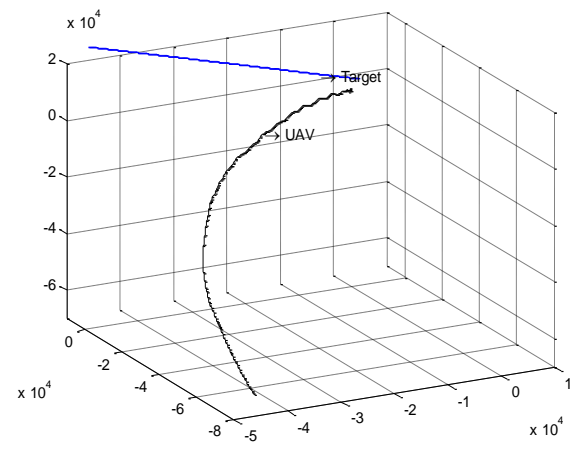

Figure 7. The Guidance Trajectories of Fuzzy Neural Network Guidance Law based on Takagi-Sugeno for UAV 
3) When the target is moving in line, guidance trajectories based on proportional guidance for UAV is shown in Figure 8 as follows:

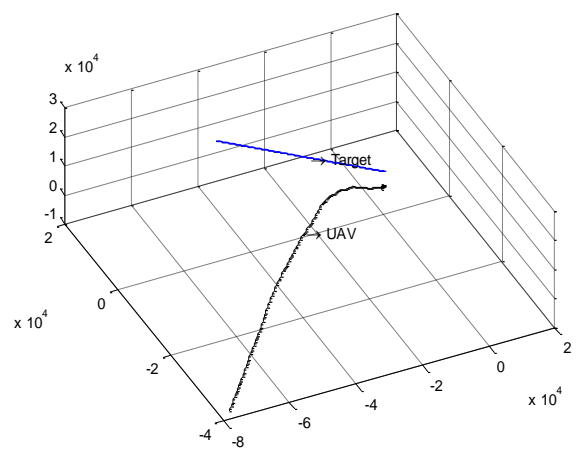

Figure 8. The Guidance Trajectories that based on Proportional Guidance for UAV

The Figure 7 and Figure 8 shows that when the target do rectilinear motion, guidance trajectory of using t-s fuzzy neural network is relatively smooth than that on proportional guidance.

4) When the target does curve movement, the guidance trajectory of fuzzy neural network guidance law which is based on Takagi-Sugeno for UAV is shown in Figure 9 as follows:

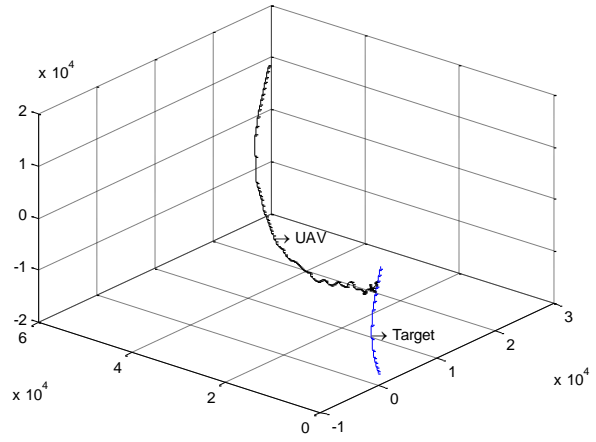

Figure 9. Trajectory of UAV Tracking Curve Moving Target

5) When the target does circle movement, the guidance trajectory of fuzzy neural network guidance law which is based on Takagi-Sugeno for UAV is shown in Figure 10 as follows: 


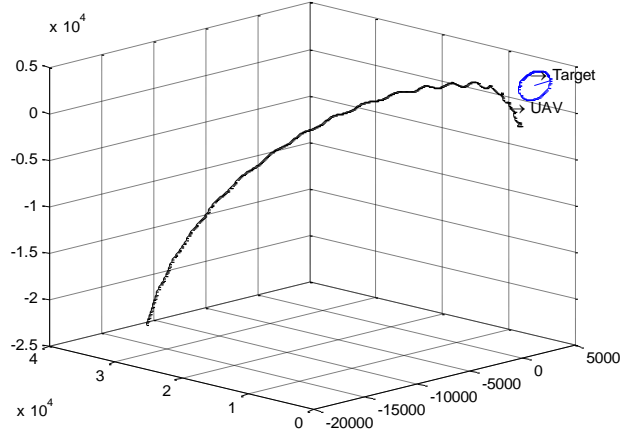

Figure 10. Trajectory of UAV Tracking Circle Moving Target

\section{Summarizes}

In this paper, we used the method of fuzzy neural network guidance law that based on Takagi-Sugeno for UAV and compared it with the proportional guidance. Seen from the simulation results, the guidance trajectory that designed in this paper is smooth and the proposed law is superior to the others .Figure 7, 8, 9 shows that this method is also suitable for target doing uniform motion in a straight line, curve movement and uniform circular motion.

\section{Acknowledgements}

I would like to appreciate teacher Yibo Li whose insightful technical comments and useful editorial suggestions improve the quality of this paper.

\section{References}

[1]. W. Xingdan and W. Honglun. UAV attacking and guidance control", The 13th annual seminar of China society of aeronautics and astronautics control and application. (2008) July, Beijing China.

[2]. C. L. Delin and S. Chunlin, "Application of RBF neural network in the optimization of guidance law", Journal of aircraft design, vol. 12, no. 4, (2006), pp. 50-53.

[3]. Z. Yunjie, C. Y. Z. Qingbin and K. HaiBo, "3-d dynamic ballistic simulation based on Matlab", vol. 24, no. 6, (2007), pp. 57-61.

[4]. O. Y. Zhonghui, L. Jiaqi, Z. Longjie and Y. Wen, "Trajectory Simulation of 3-D pure proportional guidance based on vector operation", Journal of missiles and guided journal, no. 1, (2013), pp. 53-56.

[5]. L. Zhang and K. Zhang, "Adaptive Fuzzy-PID Controllers Based on Optimal Fuzzy Reasoning for Missile Terminal Guidance”, AIAA, (2009).

[6]. Y. Kun, W. Qingxian and J. Changsheng, "Design of 3-dimensional fuzzy logic control guidance law based on PNG approaching theory", Electric and control, vol. 12, no. 6, (2007), pp. 48-52.

[7]. L. Gang, D. T. W. Yuanyuan and G. Shuqing, "BTT Missile adaptive control based on fuzzy neural network", Journal of missiles and guided journal, vol. 31, no. 1, (2011), pp. 26-28.

[8]. J. Yu, Q. Xu and Y. Zhi, "A Self-adaptive Region Fuzzy Guidance Law Based on RBF Neural Network for Attacking UAV”, IEEE, Beijing, (2011).

[9]. H. Yue, "Traffic flow forecast Based on improved t-s fuzzy neural network", Journal of computer science and exploration, vol. 8, no. 1, (2014), pp. 121-126.

[10].H. Baoru, X. Y. Liang and L. Yaoli, "Troubleshooting Analog Circuits that based on Takagi-Sugeno adaptive fuzzy neural network", Journal of electronic quality, vol. 3, (2013), pp. 31-35.

[11].Y. Hao, "The Design and Study of Dynamic Positioning Controller of Ship based on Adaptive Fuzzy Control", Dalian Maritime University, (2013).

[12].H. Xiaohong, L. Shubo and L. Yingqi, "Research of Adaptive Fuzzy Neural Network Control System", Control System, vol. 22, no. 5, (2006), pp. 85-88. 
[13].Z. Zhongshou, "The application of Fuzzy Neural Network Based on T-S model in water quality evaluation", Hohai University, (2007).

[14].L. Bingxiang, "Research on non-linear combination forecast method of enterprise financial crisis", Journal of industrial Engineering/Engineering Management, vol. 19, no. 1, (2005), pp. 19-23.

[15].S. Seong-ho, H. In-joong and A. Mester, "Lyapunov-like approach to performance analysis of 3-mensional pure PNG laws", IEEE Transactions on Aerospace and Electronic Systems, vol. 30, no. 1, (1994), pp. 238248.

[16].L. Kaicong, "The relation between fuzzy logic guidance law and homing quality", Harbin Institute of Technology, (2008).

[17].W. Sentang and F. Yuhua, "Flight control system", Beijing: Beijing University of Aeronautics and Astronautics Press, (2010).

[18]. Y. Jikai, H. Y. Bin, "Intelligent control technology”, Beijing: Beijing University of Technology Press, (1999).

[19].L. Guoyong, "Intelligent control and its MATLAB realization”, Beijing: electronic industry press, (2005).

\section{Authors}
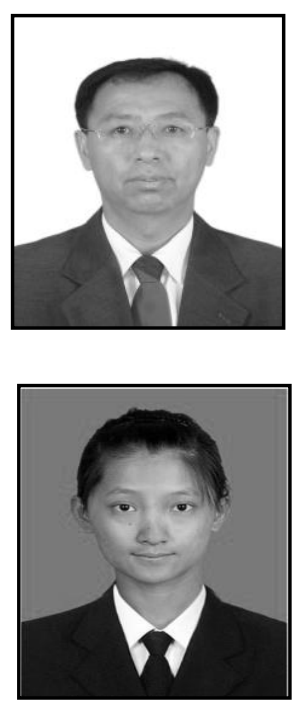

Yibo Li, Born in 1963, he received master degree from Nanjing University of Aeronautics and Astronautics in 1988 and doctor degree from Northeastern University in 2003. He became a teacher in Shenyang Aerospace University since 1988. His main research interests are image processing, pattern recognition, flight control, etc.

Zitong Li, Born in 1989, she received bachelor degree from Dalian Nationality University in 2012. Her main research interests are the research of unmanned aerial vehicle (UAV) guidance technology.

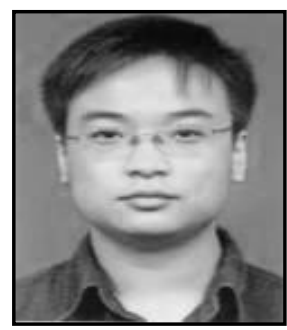

Yi Wang, Born in 1980, he received master degree from Beijing University of Aeronautics and Astronautics in 2012. He became a technical center director designer in AVIC Chengdu aircraft industrial (group) CO, LTD since 2003. His main research interests are UAV flight control and management, with flying UAV formation control, Self-control, etc.

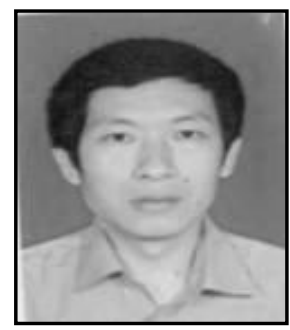

Wei Chen, Born in 1964, he received master degree from Northwestern Poly technical University in 1988. He became a chief designer in AVIC Chengdu aircraft industrial (group) CO, LTD since 1988. His main research interests are aircraft control, navigation. 